\title{
Reproduction and population dynamics of cave-dwelling bats in Costa of Oaxaca, México
}

\author{
Itandehui Hernández-Aguilar $^{1 *} \&$ Antonio Santos-Moreno ${ }^{1}$ \\ 1. Laboratorio de Ecología Animal, Centro Interdisciplinario de Investigación para el Desarrollo Integral Regional, \\ Unidad Oaxaca. Instituto Politécnico Nacional. Calle Hornos número 1003, Colonia La Noche Buena, Santa Cruz \\ Xoxocotlán, Código Postal 71230, Oaxaca, México; itandehui0901@gmail.com \\ * Correspondence
}

Received 27-VIII-2019. Corrected 13-V-2020. Accepted 19-V-2020.

\begin{abstract}
Introduction: Reproduction is a critical phase for most living organisms and in bats the reproductive strategies exhibit considerable complexity and variability. Objective: To describe the reproductive patterns and population dynamics of seven bat species (Artibeus toltecus, Carollia perspicillata, Glossophaga soricina, Mormoops megalophylla, Pteronotus fulvus, Pteronotus mesoamericanus and Natalus mexicanus) that roost in one mine (La Mina) and two caves (El Apanguito and Cerro Huatulco) in the State of Oaxaca, in Southeastern México. Methods: Sampling was conducted monthly from July 2016 to June 2017. Bats were captured using a harp trap, which was placed at the entrance of the roosts. The captured bats were identified using taxonomic keys, marked with an aluminum ring, sex and age class were also determined. Reproductive activity was modeled through 63 GLMs for each species (504 in total). The best model was selected according to the Akaike Information Criterion (AIC). Results: A total of 5836 bats were captured and marked, classified into 14 species, 10 genera, and five families. The most abundant species were: P. fulvus and P. mesoamericanus, representing $41 \%$ and $32.3 \%$ of the captures, respectively. The mormopids M. megalophylla, P. fulvus, P. mesoamerica$n u s$, together with $N$. mexicanus showed a restricted seasonal monoestrous pattern, while the phyllostomids $A$. toltecus, C. perspicillata, and G. soricina showed a seasonal bimodal polyoestry pattern. The monthly abundance of species fluctuated significantly $(\mathrm{H}=13, \mathrm{df}=11, \mathrm{P}=0.044)$ in the three roosts throughout the entire study period and the best supported GLM that included the seven species showed that the six chosen variables (season, sex, roost, temperature, precipitation and humidity) were positively associated with reproductive activity. Conclusions: The reproductive activity of each species seems to be synchronized with the end of the dry season and the beginning of the rainy season, as well as influenced by factors such as temperature, humidity and roost site.
\end{abstract}

Key words: caves; mines; monoestrous; Mormoopidae; Phyllostomidae; polyestrous; sexual segregation, Mexico.

Hernández-Aguilar, I., \& Santos-Moreno, A. (2020). Reproduction and population dynamics of cave-dwelling bats in Costa of Oaxaca, México. Revista de Biología Tropical, 68(3), $785-802$

Reproduction is a critical phase for most living organisms (Zortéa, 2003). Bats have life history strategies that are distinctive from that of mammals of similar size. Because small mammals are characterized by multiple reproductive events and, in most species, by a small litter size (one, two, and up to three offspring), where gestation and lactation are relatively prolonged (Jerrett, 1979; Racey, 1982; Racey \& Entwistle, 2000). All species of bats present care to their offspring after birth, and after weaning, females can move away from their young and feed beyond the vicinity of the refuge (Clark, Leslie, \& Carter, 1993; Burland, 1998). 
The reproductive strategies of bats exhibit considerable complexity and variability (Wilson, 1979; Racey \& Entwistle, 2000). Given that reproduction, particularly lactation, is energetically costly, the availability of food, which is directly correlated with precipitation, seems to be an important factor that determines the onset of this stage (Thompson, 1992). Therefore, in places where the food is abundant throughout the year, animals can start reproduction at any time, but in an environment dominated by seasonal changes in the climate, where the availability of food varies, certain periods of the year are more suitable for reproduction than others. Depending on the latitude, reproduction may be limited by the seasonality of the habitats. For example, in temperate zones, where the climate varies dramatically between winter and summer, hibernation plays an important role in the reproductive cycles of species (Racey, 1978; Racey, 1982). In tropical zones, although variations in temperature may not be extreme, there is a greater or lesser degree of seasonality in rainfall patterns (Racey, 1982).

In most species of tropical bats, both insectivorous and frugivorous, reproductive activity is associated with precipitation. It can act directly on the onset of reproductive activity or indirectly by its effect on the control of flowering, fruiting, and increase of populations of insects that bats feed on (Bonaccorso, 1979; Humphrey \& Bonaccorso, 1979; Racey, 1982; Cumming \& Bernard, 1997; Estrada \& CoatesEstrada, 2001a, 2001b). Births usually occur at the onset of rains and lactation occurs during the peak of this season (Racey, 1982). Bats have developed seasonal breeding patterns to ensure that both offspring and lactating mothers find favorable conditions for their survival (Racey, 1982; Altringham, 1996). Thus, they show a variety of annual reproduction patterns, which vary as much over the period of the year in which gestation occurs as in the space between them, in this way they relate to changes in climate (Jerrett, 1979). In this regard, Racey and Entwistle (2000) present ten different reproductive patterns based on the studies of Jerrett (1979) and Happold and Happold (1990). Three are monoestrous type, when there is one litter per year (restricted seasonal monoestry, extended seasonal monoestry and aseasonal monoestry). Seven other patterns are of polyestrous type, when two and three litters per year happen (seasonal bimodal polyoestry with and without postpartum oestrus, seasonal multimodal polyoestry with postpartum oestrus, continuous bimodal polyoestry with postpartum oestrus, continuous multimodal polyoestry with and without postpartum oestrus, and aseasonal polyoestry).

An important aspect in the study of bats is population dynamics since it allows evaluating future trends in their populations and therefore, knowing the conservation status and defining protection strategies (Lemos-Espinal, Rojas-González, Zúñiga-Vega, \& Jaime, 2005). Knowledge of the population structure and dynamics (age categories, sex ratio and abundance) of bats over time allows estimating the number of individuals that support the population (Lemos-Espinal et al., 2005). In cave bats that spend more than half of their lives in shelters and develop their main activities there, such as reproduction and rest, environmental conditions (temperature, humidity, air flow and light intensity) appear to be important factors in the selection of the refuge, where the population size of bat species may increase over time (Kunz, 1982; Hill \& Smith, 1984).

This study describes the reproductive patterns and population dynamics of seven bat species, two frugivorous species (Artibeus toltecus and Carollia perspicillata), four insectivorous (Mormoops megalophylla, Natalus mexicanus, Pteronotus fulvus and P. mesoamericanus) and one nectarivorous (Glossophaga soricina) in Mexico. Both Pteronotus species have also been treated as subspecies elsewhere: $P$. davyi subsp. fulvus and P. parnellii subsp. mesoamericanus, respectively. Although the reproductive patterns of these species have been studied previously in countries of America such as Costa Rica, Panamá and México (Fleming, Hooper, \& Wilson, 1972; Bateman \& Vaughan, 1974; Dinerstein, 1986; Bonaccorso, Arends, Genoud, 
Canton, \& Morton, 1992; Iñiguez-Dávalos, 1993; Ramírez-Pulido, Armella, \& CastroCampillo, 1993; Boada, Burneo, De Vries, \& Tirira, 2003; García-García, Santos-Moreno, \& Rodríguez-Alamilla, 2010 ; Torres-Flores, López-Wilchis, \& Soto-Castruita, 2012). Ecological studies of population dynamics and reproduction are relatively scarce. There is a large information gap that must be filled to understand the variability and specialization of these species throughout their distribution (Balmori, 1999). In order to contribute to knowledge in this regard, this study describes the population dynamics and reproductive patterns of the seven aforementioned species. The hypothesis proposed is that bat reproductive patterns may be related to climatic factors such as temperature and precipitation.

\section{MATERIAL AND METHODS}

Study site: The study was conducted in three roosts located in the Costa region of the State of Oaxaca, in southeastern México. The first site corresponds to a mine tunnel (15 $54^{\prime} 52^{\prime \prime} \mathrm{N} \& 96^{\circ} 24$ '59" $\mathrm{W}$ and 1110 m.a.s.l.) located in the municipality of Pluma Hidalgo. The other two sites are caves in the municipality of Santa Maria Huatulco: El Apanguito (15 51'58" N \& 96 21'13.2" $\mathrm{W}$ and 695 m.a.s.1.) and Cerro Huatulco (15'50'59" $\mathrm{N} \& 96^{\circ} 21^{\prime} 04.3^{\prime \prime} \mathrm{W}$ and 475 m.a.s.1.). The climate in the area is semi-hot subhumid with rainfall in the summer $\left(\mathrm{Aw}_{1}\right)$ (Oficina Estatal de Información para el Desarrollo Rural Sustentable [OEIDRUS], 2005), and temperature in the roosts varies throughout the year. At La Mina, the minimum temperature is 20.1 and the maximum is $23.5{ }^{\circ} \mathrm{C}$. At El Apanguito, temperature ranges between 21 and $25.7{ }^{\circ} \mathrm{C}$ and in Cerro Huatulco, it ranges from 22.3 to $26.1{ }^{\circ} \mathrm{C}$. The dominant vegetation in the area is medium subperennial rainforest with coffee plantations in the understory (Trejo, 2004; OEIDRUS, 2005).

Field work: Monthly sampling was conducted between July 2016 and June 2017 including a dry (October-April) and a rainy (May-September) season, with a sampling effort of two nights per site. Bats were captured using a harp trap (Bat Conservation and Management, Inc., Carlisle, PA, U.S.A.), 1.5 $\mathrm{m}$ wide by $2 \mathrm{~m}$ high, which was placed at the entrance of the roosts between 18:00 and 00:00 hrs. The trap was checked every $20 \mathrm{~min}$ to collect any individuals found in the trap bag. The captured bats were then placed in $15 \times 20$ $\mathrm{cm}$ cloth bags for later processing. The species of each captured individual was determined with the aid of identification keys by Medellín, Arita, and Sánchez (2007) and ÁlvarezCastañeda, Álvarez, and González-Ruiz (2015) and using the taxonomic classification system proposed by Simmons (2005). Sex and age class (young or adult) were also determined. In young males, testes are small, light colored, and covered with fur, whereas adults have larger, darker, and mostly hairless testes. Males were classified as sexually inactive or active depending on whether they had inguinal or scrotal testes, respectively. In young females, the nipples are small, light colored, and hairy, whereas in adult females, the nipples are larger, darker, and lack hair (Anthony, 1988). Females were classified as reproductively inactive if their nipples were covered with hair, as gestational when the embryo could be felt in their abdomen, and as lactating when there was milk in the nipples and these were hairless (Kunz, 1996). Finally, to avoid counting them again, each captured individual was marked on the forearm with an aluminum ring (National Band and Tag Company, Newport, Kentucky, USA) identified with a unique serial number. Rings corresponding to category A $(2.9 \mathrm{~mm})$ were used for Pteronotus fulvus and Natalus mexicanus, and category B (4 mm) rings were used for Artibeus toltecus, Carollia perspicillata, Glossophaga soricina, P. mesoamericanus, and Mormoops megalophylla. After recording the data of interest and marking individuals, they were released at their capture site. In order to carry out this study, a scientific collection license was granted for teaching purposes in the field of wildlife (20/ks-0112/10/16). 
Data analyses: The sampling effort was calculated according to the formula proposed by Medellín (1993), where the dimensions of the harp trap ( $2 \mathrm{~m}$ length, $1.5 \mathrm{~m}$ width) were multiplied by the $6 \mathrm{~h}$ it was open during 12 months of sampling (69 nights in total; 22, 24 and 23 nights in Mina, El Apanguito and Cerro Huatulco, respectively). The result was expressed as $\mathrm{m}^{2}$ net $\times \mathrm{h}$.

The classification proposed by Racey and Entwistle (2000) was followed to determine the reproductive pattern of each species. Since the largest distance between shelters is $11 \mathrm{~km}$, it was unlikely to find two different reproductive patterns in the same species. This analysis was performed for each species in each roost and there were no differences in the pattern obtained. In addition, the low abundance and records of active individuals in some species (for example, the 29 ind. captured from Mormoops megalophylla in the Cerro Huatulco cave) did not allow to identify a pattern by roost, so it was decided to combine the data of each roost per species and show a single pattern. Temperature and relative humidity data were obtained with a WM-350 WindMate ${ }^{\circledR}$ Multi-function Weather Meter (Speedtech Instruments, USA) during the field work. Monthly precipitation data were obtained from a meteorological station near the study area (Santa María Huatulco, code 20 333) provided by the National Meteorological Service (Gobierno de México, n.d.).

The significance of differences in the number of individuals captured per species and month was evaluated statistically with a non-parametric Kruskal-Wallis test (Zar, 1996). Multiple generalized linear models (GLMs) of binomial distribution $(0=$ reproductively inactive, $1=$ reproductive state) were used to investigate the relationships between the reproductive activity of the seven bats species and six explanatory variables: monthly precipitation (MPR), relative humidity (RHU), roost (ROO), season (SEA), sex (SEX) and temperature (TEM). The link function used was of the logit type. This resulted in a set of 63 possible candidate models. GLMs were run first for all species together and then for each species separately, therefore, in total 504 models were run. The best model was selected according to the Akaike Information Criterion (AIC). Analyzes were carried out in $\mathrm{R}$ programming language through the $\mathrm{R}$ Commander interface ( $\mathrm{R}$ Core Team, 2020).

\section{RESULTS}

With a sampling effort of $1242 \mathrm{~m}^{2}$ net $\times \mathrm{h}$, a total of 5836 bats from 14 species distributed in five families were captured and marked. The most abundant species were Pteronotus fulvus and $P$. mesoamericanus, representing 41 and $32.3 \%$ of the captures, respectively (Table 1 ). The monthly abundance of each species fluctuated significantly $(\mathrm{H}=13, \mathrm{df}=11, \mathrm{P}=0.044)$ throughout the entire study period (Fig. 1A, 1B, 1C). The mormopids Mormoops megalophylla, Pteronotus fulvus, $P$. mesoamericanus together with Natalus mexicanus showed a restricted seasonal monoestrous pattern while the phyllostomids Artibeus toltecus, Carollia perspicillata and Glossophaga soricina showed seasonal bimodal polyoestry pattern. Monthly abundance and reproductive patterns represent data of the three roosts evaluated.

In general, of the 63 GLMs evaluated for the seven species, the best supported model $(\mathrm{SEA}+\mathrm{MPR}+\mathrm{RHU}+\mathrm{ROO}+\mathrm{SEX}+\mathrm{TEM}, \boldsymbol{\omega}$ $=0.956)$ indicated that all the variables contribute to explain the reproductive activity of the seven bat species (Table 2). Estimated $\boldsymbol{\beta}$ coefficients indicated that the reproductive activity of the species was positively associated with females $(\boldsymbol{\beta}=2.310, \mathrm{P}<0.001)$ captured in the El Apanguito cave $(\boldsymbol{\beta}=1.501, \mathrm{P}<0.001)$ in the dry season $(\boldsymbol{\beta}=1.713, \mathrm{P}<0.001)$. The monthly precipitation was less than $100 \mathrm{~mm}(\boldsymbol{\beta}=3.569$, $\mathrm{P}<0.001)$, relative humidity between 80 $89 \%(\boldsymbol{\beta}=2.994, \mathrm{P}<0.001)$ and temperature greater than $25^{\circ} \mathrm{C}(\boldsymbol{\beta}=3.941, \mathrm{P}<0.001)$ (Table $3)$. The reproductive pattern observed in Mormoops megalophylla was restricted seasonal monoestry, with the presence of males with scrotal testes between November and February and gestation occurred between February and 
TABLE 1

Taxonomic list and abundances of the species captured in the three studied roosts

\begin{tabular}{|c|c|c|c|c|}
\hline \multirow{2}{*}{ Order Chiroptera } & \multicolumn{3}{|c|}{ Captures per roost } & \multirow{2}{*}{ Total captures } \\
\hline & La Mina & El Apanguito & Cerro Huatulco & \\
\hline \multicolumn{5}{|l|}{ Family Emballonuridae } \\
\hline Balantiopterix plicata & 0 & 0 & 1 & 1 \\
\hline \multicolumn{5}{|l|}{ Family Mormoopidae } \\
\hline Mormoops megalophylla & 0 & 816 & 29 & 845 \\
\hline Pteronotus fulvus & 2 & 1609 & 758 & 2369 \\
\hline Pteronotus mesoamericanus & 112 & 1682 & 74 & 1868 \\
\hline Pteronotus psilotis & 0 & 0 & 2 & 2 \\
\hline \multicolumn{5}{|l|}{ Family Phyllostomidae } \\
\hline Desmodus rotundus & 0 & 0 & 19 & 19 \\
\hline Glossophaga soricina & 130 & 0 & 52 & 182 \\
\hline Carollia perspicillata & 227 & 0 & 1 & 228 \\
\hline Artibeus jamaicensis & 0 & 0 & 17 & 17 \\
\hline Artibeus toltecus & 75 & 0 & 1 & 76 \\
\hline Artibeus watsoni & 7 & 0 & 0 & 7 \\
\hline Sturnira hondurensis & 7 & 0 & 1 & 8 \\
\hline \multicolumn{5}{|l|}{ Family Natalidae } \\
\hline Natalus mexicanus & 4 & 199 & 5 & 208 \\
\hline \multicolumn{5}{|l|}{ Family Vespertilionidae } \\
\hline Myotis pilosatibialis & 6 & 0 & 0 & 6 \\
\hline Total species & 9 & 4 & 12 & 14 \\
\hline Total individuals & 570 & 4306 & 960 & 5836 \\
\hline
\end{tabular}

April. This species did not use the El Apanguito cave for lactation and the colony abandoned the cave in May (Fig. 2A). No juveniles were recorded, $66.6 \%$ of adults were males, and $33.4 \%$ were females. The best model (MPR + $\mathrm{RHU}+\mathrm{ROO}+\mathrm{TEM}, \boldsymbol{\omega}=0.993)$ indicated that four of the six variables contributed to explain reproductive activity (Table 2). Also, this was positively associated with individuals captured in El Apanguito $(\boldsymbol{\beta}=1.571, \mathrm{P}<0.001)$ when the temperature was higher than $25^{\circ} \mathrm{C}(\boldsymbol{\beta}=1.752$, $\mathrm{P}<0.001)$, humidity had values of $70-79 \%$ $(\boldsymbol{\beta}=1.136, \mathrm{P}=0.003)$ and precipitation was less than $100 \mathrm{~mm}(\boldsymbol{\beta}=1.369, \mathrm{P}<0.001)$.

Pteronotus fulvus, the most abundant species at Cerro Huatulco and the second most abundant at El Apanguito (758 and 1619 individuals captured, respectively), presented a restricted seasonal monoestrous pattern. Males with scrotal testes were observed from November to February. Gestation was observed between February and April and at the beginning of the rainy season. No lactating females were recorded in Cerro Huatulco and, due to the low abundance of lactating females in El Apanguito (two in May and two in June), it is most likely that lactation occurs in another unidentified roost (Fig. 2B). The best model $(\mathrm{SEA}+\mathrm{RHU}+\mathrm{TEM}, \omega=0.961)$ indicated that three of the six variables contributed to explain the reproductive activity (Table 2 ). Estimated $\beta$ coefficients indicated that reproductive activity was positively associated with individuals caught in the dry season $(\beta=1.571, \mathrm{P}<0.001)$, when humidity was $70-79 \%(\beta=1.732, \mathrm{P}=$ $0.005)$ and the temperature higher than $25{ }^{\circ} \mathrm{C}$ $(\beta=1.935, \mathrm{P}<0.001)($ Table 3$)$.

The reproductive pattern observed in Pteronotus mesoamericanus was restricted seasonal monoestry, beginning with males with scrotal testes in November, followed by gestation starting in January and lasting until April, when 

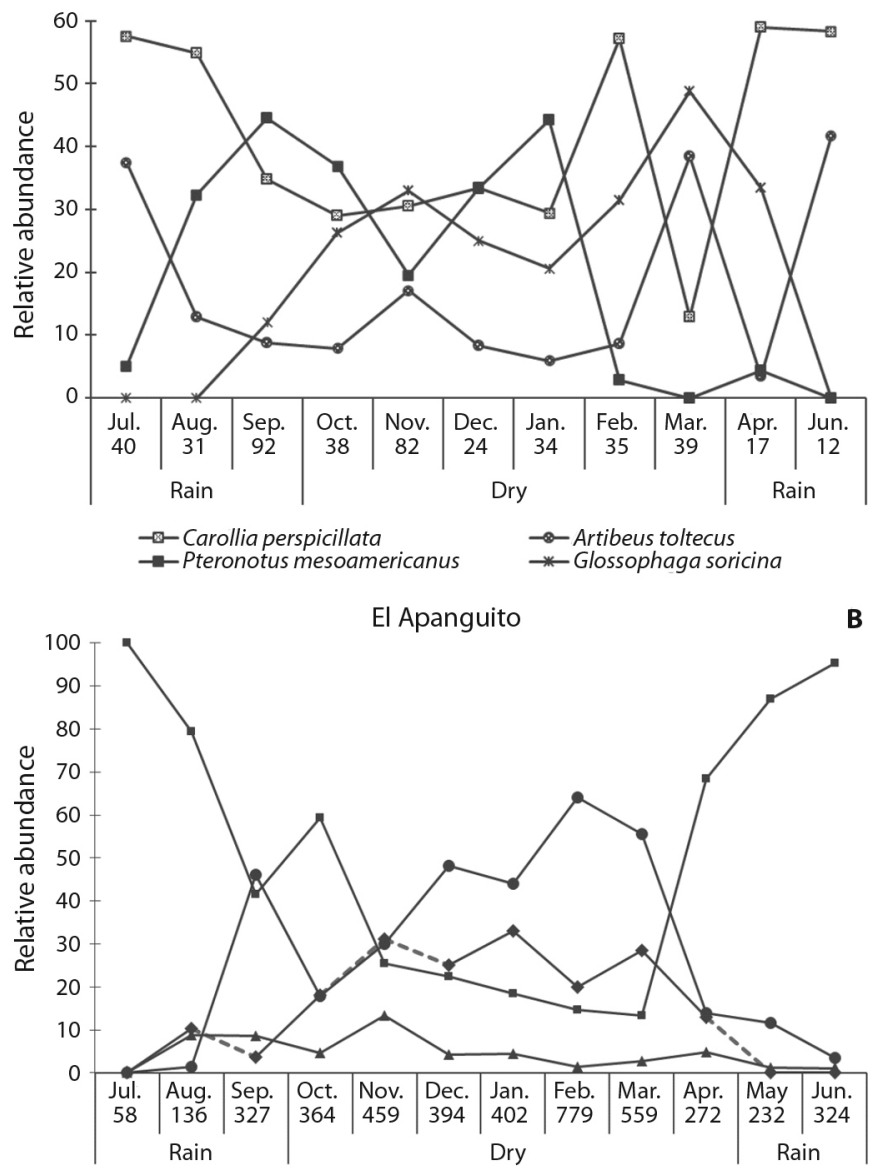

$\rightarrow$ Mormoops megalophylla $\rightarrow$ Pteronotus fulvus

$\rightarrow$ Natalus mexicanus

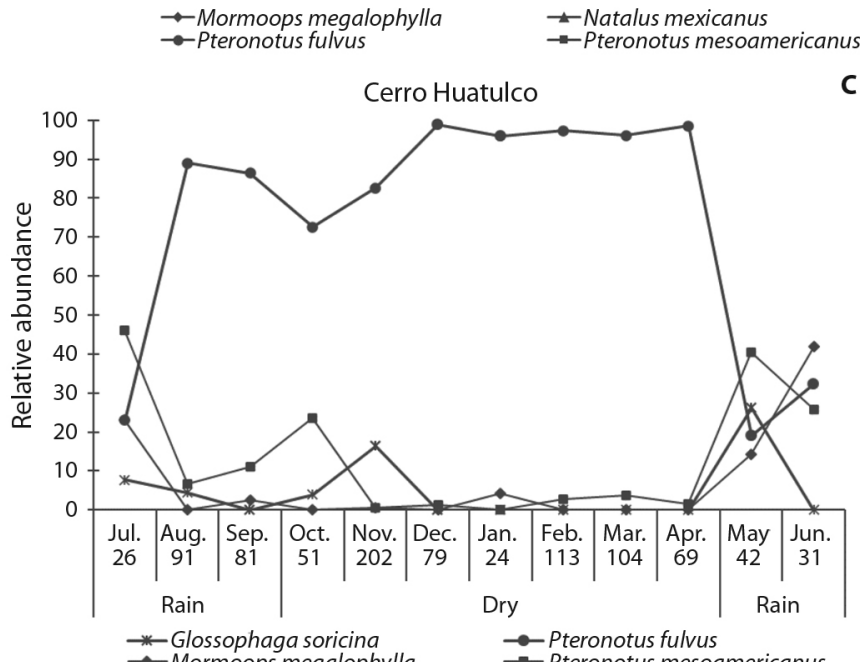

Fig. 1. Variation of relative abundance of seven bat species in three roosts found on the Costa Region of Oaxaca, Mexico. $\mathrm{X}$-axis numbers indicate the monthly captures. 
TABLE 2

Five best supported models that explain the reproductive activity of seven species of bats in Oaxaca, Mexico

\begin{tabular}{|c|c|c|c|c|}
\hline Model candidate & AIC & $\triangle \mathrm{AIC}$ & AIC Weights & Residual Deviance \\
\hline \multicolumn{5}{|l|}{ All species } \\
\hline $\mathrm{SEA}+\mathrm{MPR}+\mathrm{RHU}+\mathrm{ROO}+\mathrm{SEX}+\mathrm{TEM}$ & 3955.558 & 0.000 & 0.956 & 3915.900 \\
\hline $\mathrm{SEA}+\mathrm{RHU}+\mathrm{ROO}+\mathrm{SEX}+\mathrm{TEM}$ & 3959.888 & 4.330 & 0.110 & 3915.600 \\
\hline $\mathrm{SEA}+\mathrm{RHU}+\mathrm{SEX}+\mathrm{TEM}$ & 3980.053 & 24.445 & 0.000 & 3944.100 \\
\hline $\mathrm{SEA}+\mathrm{MPR}+\mathrm{RHU}+\mathrm{SEX}+\mathrm{TEM}$ & 3983.365 & 27.807 & 0.000 & 3954.400 \\
\hline $\mathrm{SEA}+\mathrm{MPR}+\mathrm{ROO}+\mathrm{SEX}+\mathrm{TEM}$ & 4013.237 & 57.679 & 0.000 & 3975.200 \\
\hline \multicolumn{5}{|l|}{ Artibeus toltecus } \\
\hline $\mathrm{RHU}+\mathrm{ROO}+\mathrm{SEX}+\mathrm{TEM}$ & 72.720 & 0.000 & 0.956 & 61.732 \\
\hline $\mathrm{SEA}+\mathrm{SEX}$ & 83.082 & 10.362 & 0.005 & 77.082 \\
\hline $\mathrm{SEA}+\mathrm{ROO}+\mathrm{SEX}$ & 84.000 & 11.280 & 0.003 & 76.800 \\
\hline $\mathrm{SEA}+\mathrm{RHU}+\mathrm{SEX}$ & 84.493 & 11.773 & 0.003 & 74.494 \\
\hline $\mathrm{RHU}+\mathrm{ROO}$ & 84.921 & 12.201 & 0.002 & 76.921 \\
\hline \multicolumn{5}{|l|}{ Carollia perspicillata } \\
\hline RHU + ROO+ TEM & 153.233 & 0.000 & 0.976 & 139.230 \\
\hline $\mathrm{SEA}+\mathrm{RHU}+\mathrm{ROO}+\mathrm{TEM}$ & 164.156 & 10.923 & 0.004 & 151.486 \\
\hline $\mathrm{MPR}+\mathrm{RHU}+\mathrm{ROO}+\mathrm{SEX}+\mathrm{TEM}$ & 174.641 & 21.408 & 0.000 & 162.600 \\
\hline $\mathrm{SEA}+\mathrm{RHU}+\mathrm{SEX}+\mathrm{TEM}$ & 175.395 & 22.162 & 0.000 & 165.190 \\
\hline $\mathrm{SEA}+\mathrm{MPR}+\mathrm{RHU}+\mathrm{ROO}+\mathrm{TEM}$ & 178.150 & 24.917 & 0.000 & 162.150 \\
\hline \multicolumn{5}{|l|}{ Glossophaga soricina } \\
\hline $\mathrm{MPR}+\mathrm{ROO}+\mathrm{SEX}+\mathrm{TEM}$ & 173.493 & 0.000 & 0.738 & 164.49 \\
\hline $\mathrm{SEA}+\mathrm{MPR}+\mathrm{ROO}+\mathrm{TEM}$ & 177.059 & 3.566 & 0.124 & 153.060 \\
\hline $\mathrm{SEA}+\mathrm{MPR}+\mathrm{RHU}+\mathrm{ROO}+\mathrm{TEM}$ & 178.445 & 4.962 & 0.062 & 152.450 \\
\hline $\mathrm{SEA}+\mathrm{MPR}+\mathrm{RHU}+\mathrm{TEM}$ & 178.455 & 5.539 & 0.046 & 152.550 \\
\hline $\mathrm{SEA}+\mathrm{MPR}+\mathrm{ROO}+\mathrm{SEX}+\mathrm{TEM}$ & 179.032 & 8.607 & 0.010 & 153.030 \\
\hline \multicolumn{5}{|l|}{ Natalus mexicanus } \\
\hline $\mathrm{MPR}+\mathrm{RHU}+\mathrm{ROO}+\mathrm{SEX}+\mathrm{TEM}$ & 49.047 & 0.000 & 0.905 & 27.040 \\
\hline $\mathrm{MPR}+\mathrm{RHU}+\mathrm{SEX}+\mathrm{TEM}$ & 54.541 & 5.494 & 0.058 & 42.541 \\
\hline $\mathrm{SEA}+\mathrm{MPR}+\mathrm{RHU}+\mathrm{ROO}+\mathrm{SEX}+\mathrm{TEM}$ & 56.485 & 7.438 & 0.022 & 40.885 \\
\hline $\mathrm{SEA}+\mathrm{MPR}+\mathrm{RHU}+\mathrm{SEX}+\mathrm{TEM}$ & 62.634 & 13.587 & 0.10 & 48.634 \\
\hline $\mathrm{MPR}+\mathrm{SEX}+\mathrm{TEM}$ & 62.933 & 13.886 & 0.001 & 48.002 \\
\hline \multicolumn{5}{|l|}{ Mormoops megalophylla } \\
\hline $\mathrm{MPR}+\mathrm{RHU}+\mathrm{ROO}+\mathrm{TEM}$ & 714.454 & 0.000 & 0.993 & 702.450 \\
\hline $\mathrm{MPR}+\mathrm{RHU}+\mathrm{ROO}$ & 725.480 & 11.030 & 0.004 & 709.610 \\
\hline MPR + RHU & 726.120 & 11.670 & 0.003 & 705.480 \\
\hline $\mathrm{SEA}+\mathrm{MPR}+\mathrm{RHU}+\mathrm{TEM}$ & 731.260 & 16.810 & 0.000 & 726.400 \\
\hline $\mathrm{SEA}+\mathrm{SEX}$ & 737.790 & 23.340 & 0.000 & 732.590 \\
\hline \multicolumn{5}{|l|}{ Pteronotus fulvus } \\
\hline $\mathrm{SEA}+\mathrm{RHU}+\mathrm{TEM}$ & 945.710 & 0.000 & 0.961 & 917.858 \\
\hline $\mathrm{SEA}+\mathrm{MPR}+\mathrm{RHU}+\mathrm{SEX}+\mathrm{TEM}$ & 953.040 & 7.330 & 0.025 & 935.647 \\
\hline $\mathrm{SEA}+\mathrm{MPR}+\mathrm{RHU}+\mathrm{TEM}$ & 954.525 & 8.815 & 0.012 & 938.530 \\
\hline $\mathrm{MPR}+\mathrm{RHU}+\mathrm{SEX}+\mathrm{TEM}$ & 958.050 & 12.340 & 0.002 & 938.050 \\
\hline $\mathrm{MPR}+\mathrm{RHU}+\mathrm{ROO}+\mathrm{SEX}+\mathrm{TEM}$ & 961.468 & 15.758 & 0.000 & 940.858 \\
\hline \multicolumn{5}{|l|}{ Pteronotus mesoamericanus } \\
\hline $\mathrm{MPR}+\mathrm{RHU}+\mathrm{ROO}+\mathrm{SEX}+\mathrm{TEM}$ & 1512.980 & 0.000 & 0.997 & 1491.000 \\
\hline $\mathrm{SEA}+\mathrm{MPR}+\mathrm{RHU}+\mathrm{ROO}+\mathrm{SEX}+\mathrm{TEM}$ & 1524.600 & 11.623 & 0.003 & 1502.380 \\
\hline $\mathrm{SEX}+\mathrm{MPR}+\mathrm{RHU}+\mathrm{TEM}$ & 1530.684 & 17.723 & 0.000 & 1512.720 \\
\hline $\mathrm{SEA}+\mathrm{MPR}+\mathrm{RHU}+\mathrm{SEX}+\mathrm{TEM}$ & 1532.396 & 19.423 & 0.000 & 1512.400 \\
\hline $\mathrm{MPR}+\mathrm{RHU}+\mathrm{ROO}+\mathrm{TEM}$ & 1541.520 & 28.523 & 0.000 & 1521.500 \\
\hline
\end{tabular}

MPR: monthly Precipitation, RHU: relative humidity, ROO: roost, SEA: season, SEX: Sex, TEM: temperature. The bestsupported model for each case is highlighted on top. AIC: Akaike's Information Criterion, $\triangle \mathrm{AIC}$ : differences in AIC between the respective models and the best-supported model. 
Mormoops megalophylla
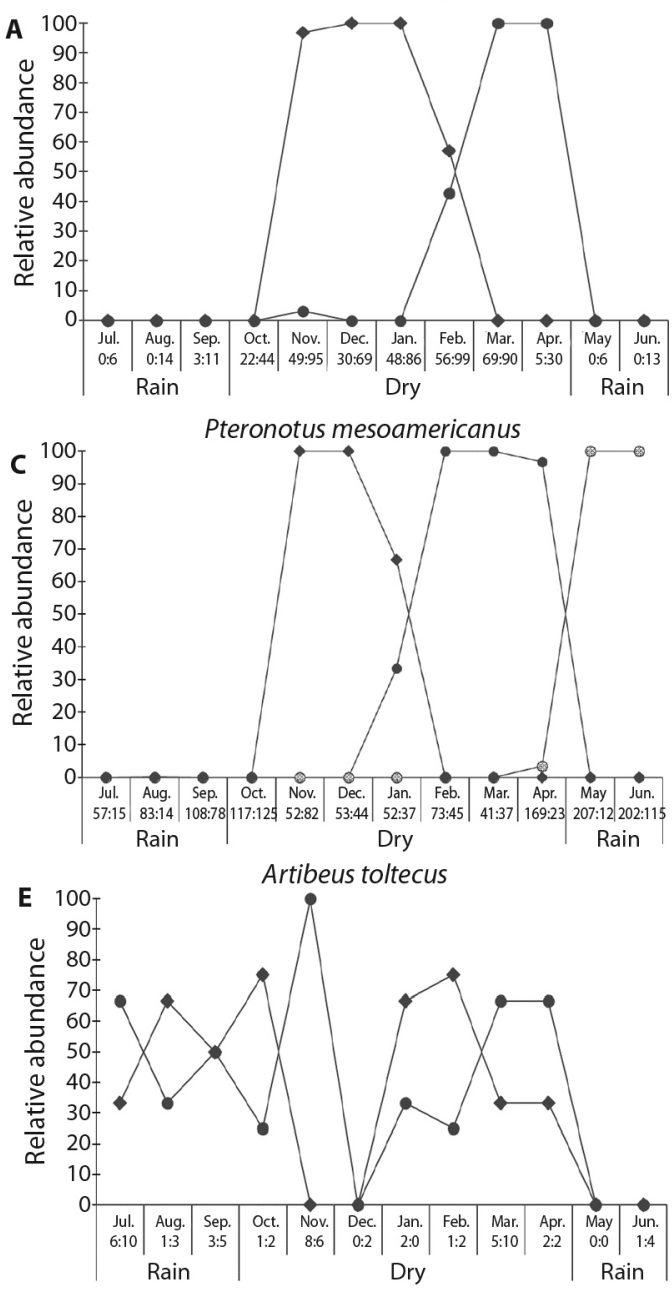

Pteronotus fulvus

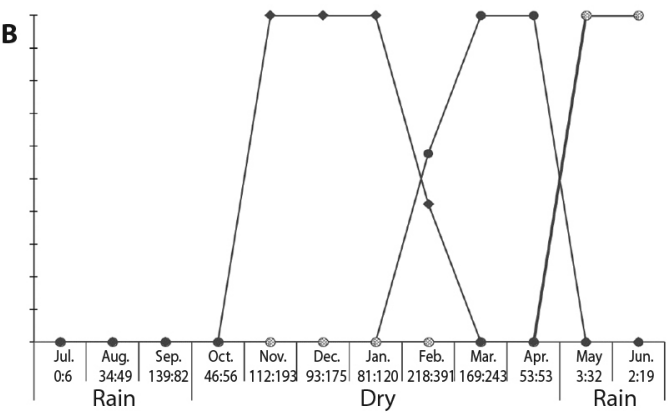

Natalus mexicanus

D
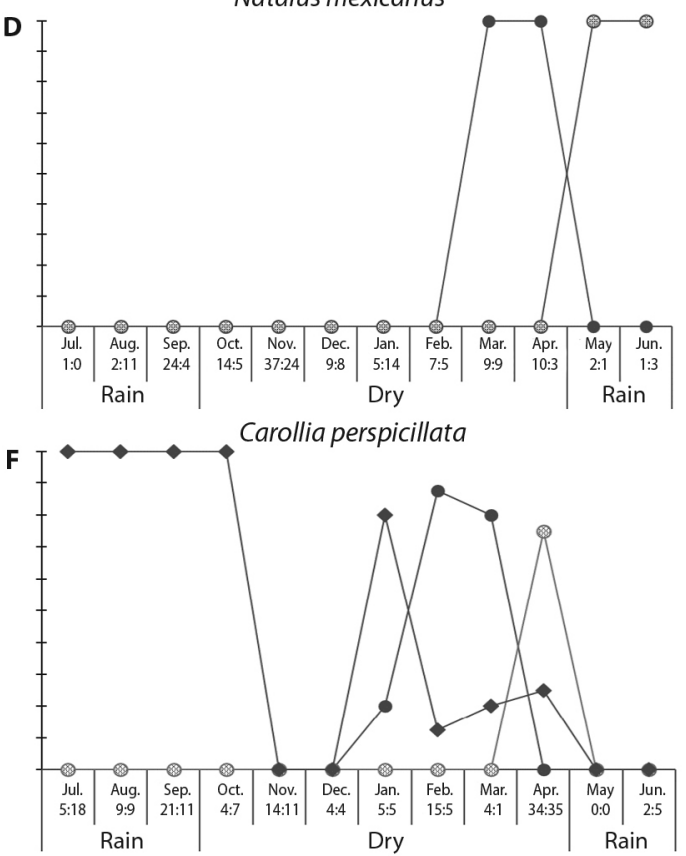

Glossophaga soricina

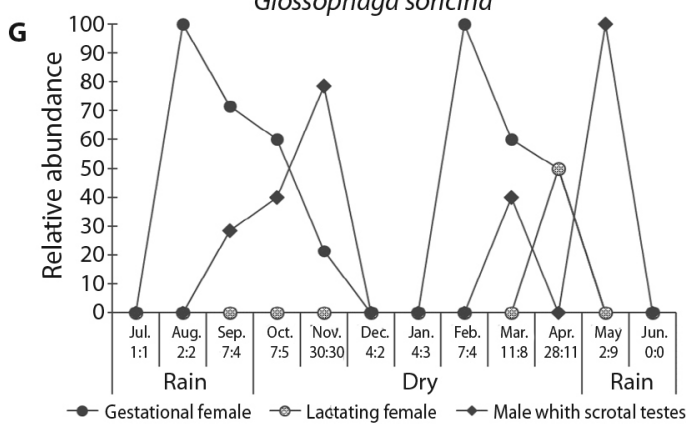

Fig. 2. Reproductive patterns of seven bat species, Costa Region of Oaxaca, Mexico. Patterns represent the data of three roosts combined. X-axis numbers indicate the ratio of adult females to adult males in the monthly captures. 
TABLE 3

Estimation of the parameters for the best-supported model that explains the reproductive activity of seven species of bats in Oaxaca, Mexico

\begin{tabular}{|c|c|c|c|c|c|}
\hline Variables & Coeficient $\boldsymbol{\beta}$ & Error & Odds-Ratio & z-value & $\mathrm{P}$ \\
\hline \multicolumn{6}{|c|}{ All species: SEA + MPR + RHU + ROO + SEX + TEM } \\
\hline Intercept & 1.805 & 16.970 & 2.691 & 0.075 & 0.940 \\
\hline \multicolumn{6}{|l|}{ Season } \\
\hline Dry & 1.713 & 0.338 & 3.235 & 0.650 & $<0.001$ \\
\hline Rainy & 0.961 & 0.356 & 1.117 & 0.058 & 0.005 \\
\hline \multicolumn{6}{|l|}{ Monthly Precipitation } \\
\hline$<100 \mathrm{~mm}$ & 3.569 & 0.658 & 6.831 & 0.513 & $<0.001$ \\
\hline $101-200 \mathrm{~mm}$ & -9.591 & 196.969 & -0.031 & -0.049 & 0.961 \\
\hline$>201 \mathrm{~mm}$ & 1.507 & 0.843 & 2.162 & 0.179 & 0.003 \\
\hline \multicolumn{6}{|l|}{ Relative Humidity } \\
\hline$<69 \%$ & 0.036 & 0.189 & 0.837 & 0.194 & 0.846 \\
\hline $70-79 \%$ & 0.889 & 0.297 & 4.110 & 2.989 & 0.002 \\
\hline $80-89 \%$ & 2.924 & 0.418 & 5.370 & 6.981 & $<0.001$ \\
\hline$>90 \%$ & 1.367 & 0.317 & 4.632 & 3.110 & 0.003 \\
\hline \multicolumn{6}{|l|}{ Roost } \\
\hline Mine & 0.763 & 0.170 & 2.660 & 3.483 & 0.001 \\
\hline Cave Cerro Huatulco & 0.769 & 0.256 & 2.912 & 3.001 & 0.001 \\
\hline Cave El Apanguito & 1.501 & 0.147 & 6.057 & 4.404 & $<0.001$ \\
\hline \multicolumn{6}{|l|}{ Sex } \\
\hline Female & 2.310 & 0.514 & 4.541 & 5.32 & $<0.001$ \\
\hline Male & 0.696 & 0.085 & 2.983 & 3.125 & 0.001 \\
\hline \multicolumn{6}{|l|}{ Temperature } \\
\hline $15-19^{\circ} \mathrm{C}$ & 1.741 & 0.374 & 0.991 & 8.042 & 0.008 \\
\hline $20-24^{\circ} \mathrm{C}$ & 1.156 & 0.517 & 0.367 & 8.012 & 0.028 \\
\hline$>25{ }^{\circ} \mathrm{C}$ & 3.941 & 0.490 & 7.777 & 9.378 & $<0.001$ \\
\hline \multicolumn{6}{|c|}{ Artibeus toltecus: RHU + ROO + SEX + TEM } \\
\hline Intercept & 2.107 & 1.472 & 8.226 & 1.431 & 0.152 \\
\hline \multicolumn{6}{|l|}{ Relative Humidity } \\
\hline $70-79 \%$ & 0.683 & 0.613 & 0.006 & 1.113 & 0.265 \\
\hline $80-89 \%$ & 1.807 & 1.839 & 1.505 & 0.311 & 0.003 \\
\hline \multicolumn{6}{|l|}{ Roost } \\
\hline Mine & - & - & - & - & - \\
\hline \multicolumn{6}{|l|}{ Sex } \\
\hline Female & 1.031 & 0.596 & 1.537 & 1.728 & 0.004 \\
\hline Male & 0.619 & 0.458 & 0.356 & 0.156 & 0.084 \\
\hline \multicolumn{6}{|l|}{ Temperature } \\
\hline $15-19^{\circ} \mathrm{C}$ & -0.959 & 6.180 & 0.001 & -0.005 & 0.996 \\
\hline $20-24^{\circ} \mathrm{C}$ & 2.401 & 1.328 & 7.090 & 1.808 & $<0.001$ \\
\hline \multicolumn{6}{|c|}{ Carollia perspicillata: RHU + ROO+ TEM } \\
\hline Intercept & -0.221 & 0.977 & 0.801 & -0.226 & 0.821 \\
\hline \multicolumn{6}{|l|}{ Relative Humidity } \\
\hline $70-79 \%$ & 1.044 & 0.295 & 0.002 & 0.012 & 0.790 \\
\hline $80-89 \%$ & 1.584 & 0.481 & 1.502 & 3.294 & 0.005 \\
\hline \multicolumn{6}{|l|}{ Roost } \\
\hline Mine & - & - & - & - & - \\
\hline
\end{tabular}


TABLE 3 (Continued)

\begin{tabular}{|c|c|c|c|c|c|}
\hline Variables & Coeficient $\boldsymbol{\beta}$ & Error & Odds-Ratio & z-value & $\mathrm{P}$ \\
\hline \multicolumn{6}{|l|}{ Temperature } \\
\hline $15-19^{\circ} \mathrm{C}$ & 0.584 & 0.571 & 0.205 & 2.772 & 0.596 \\
\hline $20-24^{\circ} \mathrm{C}$ & 1.760 & 6.180 & 3.201 & 0.004 & 0.005 \\
\hline \multicolumn{6}{|c|}{ Glossophaga soricina: MPR + ROO + SEX + TEM } \\
\hline Intercept & 3.7597 & 1.0651 & 42.934 & 3.530 & 0.0004 \\
\hline \multicolumn{6}{|l|}{ Monthly Precipitation } \\
\hline$<100 \mathrm{~mm}$ & 1.073 & 0.565 & 1.300 & 5.437 & $<0.001$ \\
\hline$>201 \mathrm{~mm}$ & 0.865 & 0.634 & 0.046 & 2.733 & 0.003 \\
\hline \multicolumn{6}{|l|}{ Roost } \\
\hline Mine & 1.891 & 0.476 & 2.118 & 0.056 & $<0.001$ \\
\hline Cave Cerro Huatulco & 1.328 & 0.321 & 0.409 & 0.870 & 0.061 \\
\hline \multicolumn{6}{|l|}{ Sex } \\
\hline Female & 1.050 & 0.731 & 1.266 & 0.160 & 0.004 \\
\hline Male & 0.604 & 0.402 & 0.666 & 0.354 & 0.872 \\
\hline \multicolumn{6}{|l|}{ Temperature } \\
\hline $15-19^{\circ} \mathrm{C}$ & -1.631 & 0.562 & 0.275 & -2.790 & 0.737 \\
\hline $20-24^{\circ} \mathrm{C}$ & 1.914 & 0.685 & 6.147 & 2.795 & $<0.001$ \\
\hline$>25^{\circ} \mathrm{C}$ & -4.485 & 1.353 & 0.011 & -3.313 & 0.549 \\
\hline \multicolumn{6}{|c|}{ Natalus mexicanus: MPR + RHU + ROO + SEX + TEM } \\
\hline Intercept & 12.046 & 8.397 & 1.703 & 0.000 & 0.999 \\
\hline \multicolumn{6}{|l|}{ Monthly Precipitation } \\
\hline$<100 \mathrm{~mm}$ & 2.534 & 0.381 & 9.078 & 0.006 & $<0.001$ \\
\hline$>201 \mathrm{~mm}$ & 0.476 & 0.839 & 0.878 & 0.136 & 0.691 \\
\hline \multicolumn{6}{|l|}{ Relative Humidity } \\
\hline $70-79 \%$ & 0.269 & 0.067 & 0.565 & 0.001 & 0.299 \\
\hline $80-89 \%$ & 2.793 & 1.024 & 3.163 & 2.729 & 0.004 \\
\hline$>90 \%$ & 0.046 & 1.604 & 0.009 & 1.213 & 0.424 \\
\hline \multicolumn{6}{|l|}{ Roost } \\
\hline Mine & -0.121 & 0.680 & 0.266 & -0.002 & 0.499 \\
\hline Cave Cerro Huatulco & -0.621 & 0.111 & 0.657 & -0.645 & 0.689 \\
\hline Cave El Apanguito & 3.163 & 0.253 & 8.715 & 0.741 & $<0.001$ \\
\hline \multicolumn{6}{|l|}{ Sex } \\
\hline Female & 1.999 & 0.841 & 10.004 & 0.568 & $<0.001$ \\
\hline Male & -3.974 & 0.452 & 0.022 & -0.004 & 0.996 \\
\hline \multicolumn{6}{|l|}{ Temperature } \\
\hline $15-19^{\circ} \mathrm{C}$ & -0.613 & 0.643 & 0.232 & -0.037 & 0.729 \\
\hline $20-24^{\circ} \mathrm{C}$ & -0.120 & 0.216 & 0.032 & -0.405 & 0.621 \\
\hline$>25{ }^{\circ} \mathrm{C}$ & 1.526 & 0.742 & 9.235 & 0.655 & $<0.001$ \\
\hline \multicolumn{6}{|c|}{ Mormoops megalophylla: MPR + RHU + ROO + TEM } \\
\hline Intercept & 2.061 & 1.164 & 1.191 & 0.028 & 0.978 \\
\hline \multicolumn{6}{|l|}{ Monthly Precipitation } \\
\hline$<100 \mathrm{~mm}$ & 1.369 & 0.039 & 4.358 & 0.586 & $<0.001$ \\
\hline $101-200 \mathrm{~mm}$ & -0.791 & 0.930 & 0.358 & -0.026 & 0.878 \\
\hline \multicolumn{6}{|l|}{ Relative Humidity } \\
\hline$<69 \%$ & 0.604 & 0.256 & 0.546 & 2.363 & 0.189 \\
\hline $70-79 \%$ & 1.136 & 0.578 & 2.970 & 0.856 & 0.003 \\
\hline $80-89 \%$ & 0.143 & 0.215 & 0.866 & 0.664 & 0.506 \\
\hline$>90 \%$ & -1.387 & 9.4352 & 0.639 & -0.020 & 0.984 \\
\hline
\end{tabular}


TABLE 3 (Continued)

\begin{tabular}{|c|c|c|c|c|c|}
\hline Variables & Coeficient $\boldsymbol{\beta}$ & Error & Odds-Ratio & z-value & $\mathrm{P}$ \\
\hline \multicolumn{6}{|l|}{ Roost } \\
\hline Cave Cerro Huatulco & -0.567 & 9.4345 & 0.580 & 0.019 & 0.984 \\
\hline Cave El Apanguito & 1.571 & 0.356 & 6.658 & 0.587 & $<0.001$ \\
\hline \multicolumn{6}{|l|}{ Temperature } \\
\hline $15-19^{\circ} \mathrm{C}$ & -0.186 & 0.367 & 0.003 & -0.005 & 0.932 \\
\hline $20-24^{\circ} \mathrm{C}$ & 0.612 & 0.479 & 1.346 & 0.343 & 0.005 \\
\hline$>25^{\circ} \mathrm{C}$ & 1.752 & 0.742 & 2.235 & 0.555 & $<0.001$ \\
\hline \multicolumn{6}{|c|}{ Pteronotus mesoamericanus: MPR + RHU + ROO + SEX + TEM } \\
\hline Intercept & -8.952 & 3.646 & 0.0001 & -0.025 & 0.980 \\
\hline \multicolumn{6}{|l|}{ Monthly Precipitation } \\
\hline$<100 \mathrm{~mm}$ & 12.220 & 3.646 & 4.258 & 0.034 & $<0.001$ \\
\hline$>201 \mathrm{~mm}$ & 7.223 & 3.635 & 1.371 & 0.020 & 0.984 \\
\hline \multicolumn{6}{|l|}{ Relative Humidity } \\
\hline$<69 \%$ & 0.559 & 0.293 & 0.571 & 1.908 & 0.0564 \\
\hline $70-79 \%$ & 0.887 & 0.243 & 0.775 & 2.310 & 0.0427 \\
\hline $80-89 \%$ & 1.191 & 0.212 & 3.290 & 5.597 & $<0.001$ \\
\hline$>90 \%$ & 1.909 & 0.222 & 6.746 & 8.582 & $<0.001$ \\
\hline \multicolumn{6}{|l|}{ Roost } \\
\hline Mine & -1.297 & 0.403 & 0.271 & -3.217 & 0.001 \\
\hline Cave Cerro Huatulco & -1.294 & 0.428 & 0.274 & -3.022 & 0.002 \\
\hline Cave El Apanguito & 1.976 & 0.273 & 6.367 & 6.285 & $<0.001$ \\
\hline \multicolumn{6}{|l|}{ Sex } \\
\hline Female & 1.870 & 0.378 & 5.380 & 0.112 & $<0.001$ \\
\hline Male & -0.834 & 0.154 & 0.434 & -5.406 & 0.001 \\
\hline \multicolumn{6}{|l|}{ Temperature } \\
\hline $15-19^{\circ} \mathrm{C}$ & 0.932 & 0.378 & 0.187 & 6.659 & 0.059 \\
\hline $20-24^{\circ} \mathrm{C}$ & 0.686 & 0.540 & 0.933 & 10.517 & 0.067 \\
\hline$>25^{\circ} \mathrm{C}$ & 3.917 & 0.452 & 3.019 & 8.660 & $<0.001$ \\
\hline \multicolumn{6}{|c|}{ Pteronotus fulvus: SEA + RHU + TEM } \\
\hline Intercept & 3.917 & 27.980 & 0.502 & 0.000 & 0.999 \\
\hline \multicolumn{6}{|l|}{ Season } \\
\hline Dry & 1.432 & 0.545 & 5.774 & 0.687 & $<0.001$ \\
\hline Rainy & 0.017 & 0.456 & 1.117 & 0.589 & 0.675 \\
\hline \multicolumn{6}{|l|}{ Relative Humidity } \\
\hline$<69 \%$ & 0.235 & 0.284 & 0.789 & 0.828 & 0.407 \\
\hline $70-79 \%$ & 1.732 & 0.578 & 2.456 & 0.678 & 0.005 \\
\hline $80-89 \%$ & 1.297 & 2.501 & 1.055 & 0.001 & 0.005 \\
\hline$>90 \%$ & -2.434 & 1.339 & 0.201 & -0.024 & 0.980 \\
\hline \multicolumn{6}{|l|}{ Temperature } \\
\hline $15-19^{\circ} \mathrm{C}$ & -1.913 & 0.253 & 0.049 & -0.002 & 0.918 \\
\hline $20-24^{\circ} \mathrm{C}$ & -2.451 & 0.798 & 0.424 & -0.001 & 0.939 \\
\hline$>25^{\circ} \mathrm{C}$ & 1.935 & 0.465 & 8.233 & 0.677 & $<0.0001$ \\
\hline
\end{tabular}


it peaked (77\%). The first births occurred also in April and increased with the beginning of the rainy season in May and June (Fig. 2C). Captured individuals were mostly adults $(98.6 \%)$. The best model (MPR + RHU + ROO + SEX + TEM, $\omega=0.997)$ indicated that five of the six variables contributed to explain reproductive activity (Table 2 ). Reproductive activity was positively associated with females $(\beta=1.870, \mathrm{P}$ $<0.001)$ captured in the El Apanguito cave $(\beta=$ $1.976, \mathrm{P}<0.001)$, when temperature was higher than $25{ }^{\circ} \mathrm{C}(\beta=3.917, \mathrm{P}<0.001)$, monthly precipitation less than $100 \mathrm{~mm}(\beta=12.220, \mathrm{P}<$ 0.001 ) and relative humidity greater than $90 \%$ $(\beta=1.909, \mathrm{P}<0.001)$ (Table 3).

Natalus mexicanus presented a restricted seasonal monoestrous pattern beginning in March and April, with the occurrence of gestating females, followed by lactating females in May and June. There were no records of males with scrotal testes (Fig. 2D). All captured individuals were adults, except one juvenile individual, which was observed in June. The best model (MPR + RHU + ROO + SEX + TEM, $\omega=0.905$ ) indicated that five of the six variables contributed to explain reproductive activity (Table 2). It was positively associated with females $(\boldsymbol{\beta}=1.999, \mathrm{P}<0.001)$ captured in El Apanguito $(\boldsymbol{\beta}=3.163, \mathrm{P}<0.001)$, when humidity presented values between $80-89$ $\%(\boldsymbol{\beta}=2.793, \mathrm{P}=0.004)$, the temperature was higher than $25^{\circ} \mathrm{C}(\boldsymbol{\beta}=1.526, \mathrm{P}<0.001)$ and precipitation less than $100 \mathrm{~mm}(\boldsymbol{\beta}=2.534, \mathrm{P}<$ 0.001) (Table 3).

Artibeus toltecus, a phyllostomid bat, showed seasonal bimodal polyoestry pattern. The first reproductive period was observed from July to November and the second between January and April. Gestating females were observed of July to October, and November, and January-April. Males with scrotal testes were recorded from July to October and from January to April (Fig. 2E). The species did not use any roosts for lactation. Adult individuals comprised $88 \%$ of captures and all juveniles were recorded in March. The best model (RHU + ROO + SEX + TEM, $\omega=0.956)$ indicated that four of the six variables contributed to explain reproductive activity (Table 2 ). Reproductive activity was positively associated with females $(\beta=1.031, \mathrm{P}=0.004)$, when the relative humidity presented values between $80-89 \%$ $(\beta=1.807, P=0.003)$ and the temperature of 20 $-24{ }^{\circ} \mathrm{C}(\beta=2.401, \mathrm{P}<0.001)$ (Table 3$)$.

Carollia perspicillata male data suggested a seasonal bimodal polyoestry pattern. In the first period, active males were only recorded from July to October and no pregnant or lactating females were recorded in this period. The second period was from January to April. Males with scrotal testes were recorded from January to April and gestating and lactating females were observed from January to April (Fig. 2F). Adult individuals represented $82 \%$ of the captures and the rest were juveniles, 97 $\%$ of which were recorded in April. The best model $(\mathrm{RHU}+\mathrm{ROO}+\mathrm{TEM}, \omega=0.976)$ indicated that three of the six variables contributed to explain reproductive activity (Table 2 ). The estimated $\beta$ coefficients indicated that this is positively associated when the relative humidity presents values between $80-89 \%(\beta=$ $1.584, \mathrm{P}=0.005)$ and a temperature of $20-24$ ${ }^{\circ} \mathrm{C}(\beta=1.760, \mathrm{P}=0.005)$ (Table 3$)$.

Glossophaga soricina showed seasonal bimodal polyoestry pattern with two reproductive periods. The first occurred between August and November, and the second was observed from February to May. Males with scrotal testes were recorded from September to November, March and May. Gestating females were observed from August to November and from February to April (Fig. 2G). Adults comprised $75 \%$ of the captures. The best model (MPR + ROO + SEX + TEM, $\omega=0.738)$ indicated that four of the six variables contributed to explain reproductive activity (Table 2). Estimated $\beta$ coefficients indicated that this was positively associated with females $(\beta=1.050, \mathrm{P}=0.004)$ captured in the Mine $(\beta=1.891, \mathrm{P}<0.001)$, when the precipitation was less than $100 \mathrm{~mm}$ $(\beta=1.073, P<0.001)$ and the temperature of $20-24{ }^{\circ} \mathrm{C}(\beta=1.914, \mathrm{P}<0.001)($ Table 3$)$. 


\section{DISCUSSION}

According to the results obtained and the hypotheses proposed, the monthly abundances of bat species varied between shelters (except Glossophaga soricina) and only for Mormoops megalophylla and Pteronotus fulvus between seasons. In addition, for the seven species of bats, at least one reproductive pattern was identified. Bat species varied in the time of the beginning and the duration of their reproductive periods. The reproductive activity of the species is influenced by the six variables selected in the GLMs (season, sex, roost, temperature, precipitation and relative humidity), highlighting the temperature, which was present in the best model of the seven species studied, while humidity and precipitation were present in the models of six and four species, respectively. The roost, except for P. fulvus, also influences the reproductive activity of all the species of bats studied. Furthermore, in the best supported model of all the species and separately, the probability of reproductive activity was higher for females captured in El Apanguito or La Mina in the dry season (October-April), when the precipitation is less than $100 \mathrm{~mm}$. Therefore, in bat species the beginning and duration of their reproductive periods will vary according to climatic factors and ecological characteristics of the species and its roosts.

In general, except Artibeus toltecus and Natalus mexicanus, the highest abundance of males with scrotal testes occurred in the middry season, from November to January. Gestating females were observed at the end of the dry season, while births occurred at the beginning of the rainy season, in May, June and July. Reproductive activity may be synchronized with the beginning of the rainy season, mainly in monoestrous species, although in bimodal polyestrous species, at least one reproductive period coincides with the rainy season when there is a higher abundance of food resources available for lactating females that have increased energy requirements, as has been previously suggested by some authors
(Fleming et al., 1972; Racey, 1982; Dinerstein, 1986; Estrada \& Coates-Estrada, 2001a). This reproductive strategy has been observed in species which base their diet on fruits and insects (Bradbury \& Veherencamp, 1976; Racey, 1982; Cumming \& Bernard, 1997; Racey \& Entwistle, 2000).

The influence of seasonality may not be so marked mainly in species that have more than one estrous cycle per year, that is, species that show reproductive activity during most of the year, where it has also been observed that the peaks of reproductive activity do not coincide significantly with the periods of greater food availability (for example C. perspicillata). This could be due to the type of food that the species consumes. For example, in $C$. perspicillata the main and most important element in its diet are fruits of Piper and Solanum, which bear fruit throughout the year (Fleming, 1991; Estrada, Coates-Estrada, \& Meritt, 1993). In addition, other authors have suggested that this species rather presents a generalist strategy, that is, it is able to consume other types of resources that are available during a particular season, being able to extend its diet to insects when the availability of Piper fruit is low (Mello, Schittini, Selig, \& Bergallo, 2004a, 2004b).

Other important factors in bat reproductive activity, according to the GLMs, were relative humidity and temperature. In phyllostomids the probability of reproductive activity is higher when the relative humidity is $80-89 \%$ and the temperature is $20-24{ }^{\circ} \mathrm{C}$, while in mormopids it occurs at temperatures higher than $25^{\circ} \mathrm{C}$ and lower relative humidity ( $70-79 \%$ ), in the case of Pteronotus fulvus and Mormoops megalophylla, and higher $(90 \%)$ for P. mesoamericanus. These conditions appear in the months of May and June and the most evident changes could be observed in El Apanguito, where the formation of maternity colonies of thousands of females of $P$. mesoamericanus, $P$. fulvus, and $N$. mexicanus was observed. In April, females of $P$. mesoamericanus dominated the roost, representing $90 \%$ of the individuals present in the cave. In the same month, females coming from different sites congregated to 
complete gestation and the care and development of offspring (we corroborated through recaptures that some females came from both La Mina and Cerro Huatulco); whereas males of $P$. mesoamericanus leave the cave, showing segregation of sexes during this period. This behavior has been reported in Sinaloa (Bateman \& Vaughan, 1974) and Colima in Mexico (Torres-Flores et al., 2012).

The restricted seasonal monoestry pattern observed in Pteronotus fulvus agrees with that reported by other authors (Wilson, 1973; Bateman \& Vaughan, 1974; Jiménez-Guzmán $\&$ Ceballos, 2005). Due to the low number of recorded lactating females $(0.2 \%$ in El Apanguito and absent in Cerro Huatulco), it can be inferred that lactation takes place in an alternative roost and is likely to occur between May and September, as has been reported by other authors in Mexico (Adams, 1989; JiménezGuzmán \& Ceballos, 2005; Torres-Flores et al., 2012). According to the study conducted by Torres-Flores et al. (2012) in Cueva El Salitre, Colima, Mexico, there are variations in time and duration of the reproductive period. In this study, reproduction occurred from December to January as shown by males with scrotal testes and gestational females from February to April, while in El Salitre active males were recorded in January-April, September, and November-December and gestational females in August-November and March-November. These variations could be explained by adjustments in the reproductive strategies of the species according to the habitat, since in El Salitre the habitat is a relict of lowland deciduous forest, while in this study it is subperennifolia medium forest.

The restricted seasonal monoestry pattern observed in Mormoops megalophylla and Natalus mexicanus also agrees with that reported in other studies (Sánchez-Hernández, ChavezTapia, Nunez-Garduño, Ceballos-Corona, \& Gurrola-Hidalgo, 1985; Bonaccorso et al., 1992; Rezsutek \& Cameron, 1993; Boada et al., 2003; Torres-Flores et al., 2012). In the case of M. megalophylla, it was possible to corroborate through individuals marked in El
Apanguito and recaptured in Cerro Huatulco, that the species presents sexual segregation during lactation. In May, females leave El Apanguito and move to another unidentified roost, while males go to Cerro Huatulco (colony formed exclusively by males). This behavior has been reported in Venezuela (Bonaccorso et al., 1992), Ecuador (Boada et al., 2003), and México (Torres-Flores et al., 2012). Although variations have been observed in dry forests where M. megalophylla does not completely abandon the roost, which, according to the hypothesis proposed by Torres-Flores et al. (2012), could indicate an adjustment in reproductive strategies according to habitat, food availability or competition.

Torres-Flores et al. (2012) mentioned that when the reproductive period of the species begins in a refuge, the most abundant species will tend to use a larger perching area and will displace others whose abundance decreases drastically. This behavior could be observed in Pteronotus mesoamericanus in El Apanguito, where abundances increase considerably from March, with the arrival of pregnant females that cover a greater perching area. This may be why M. megalophylla, P. fulvus, and $N$. mexicanus are forced to leave the cave El Apanguito in order to carry out parturition and lactation in other alternative roost. However, the abandonment of the cave by these species could be related to their physiological requirements during the breeding season, because the best GLM model supported for this species $(\mathrm{MPR}+\mathrm{RHU}+\mathrm{ROO}+\mathrm{TEM})$ indicated that reproductive activity is mainly favored by environmental factors such as temperature ( $>$ $\left.25{ }^{\circ} \mathrm{C}\right)$, precipitation $(<100 \mathrm{~mm})$ and relative humidity (70 - 79 \%). In May, when El Apanguito cave is completely abandoned, the temperature was $21^{\circ} \mathrm{C}$ and $92 \%$ relative humidity. Probably these unsuitable characteristics are what force the species to move to other sites. In addition, it is known that Mormopids have a preference for shelters with temperatures higher than $30{ }^{\circ} \mathrm{C}$ in other roosts (Rezsutek \& Cameron, 1993; Ávila-Flores \& Medellín, 2004; Torres-Flores \& Santos-Moreno, 2017; 
Ayala-Téllez, Iñiguez-Dávalos, Olvera-Vargas, Vargas-Contreras, \& Herrera-Lizaola, 2018). Maximum temperatures are not higher than 27 ${ }^{\circ} \mathrm{C}$ in any of the shelters observed in this study. Therefore, it would be useful to investigate the variations in the environmental characteristics of the shelters, as well as the specific requirements of each species in subsequent studies to corroborate these hypotheses.

The seasonal bimodal polyoestry pattern observed in the phyllostomids Artibeus toltecus, Carollia perspicillata and Glossophaga soricina agrees with reports from México (Michoacán, Sánchez-Hernández et al., 1985; Jalisco, Iñiguez-Dávalos, 1993; Guerrero, Almazán-Catalán, Sánchez-Hernández, Romero-Almaraz, Sánchez-Vasquez, \& GonzálezPérez, 2015; Ramírez-Pulido et al., 1993; Oaxaca, García-García et al., 2010; Colima, Torres-Flores et al., 2012), Costa Rica (Fleming et al., 1972; Dinerstein, 1986), Panama (Fleming et al., 1972), and Brazil (Mello \& Fernández, 2000). The best supported GLMs of the three species showed temperature and roost as important variables in common (A. toltecus: $\mathrm{RHU}+\mathrm{ROO}+\mathrm{SEX}+\mathrm{TEM}, C$. perspicillata: $\mathrm{RHU}+\mathrm{ROO}+\mathrm{TEM}$, G. soricina: MPR + $\mathrm{ROO}+\mathrm{SEX}+\mathrm{TEM}$ ), while the precipitation was only for G. soricina and the temporality for none of the three.

This reproductive pattern, seasonal bimodal polyoestry, is characterized by two estrous cycles per year, one at the end of the dry season (March-April) and another one at the end of the rainy season (July-August) (Hill \& Smith, 1984). In the populations studied, this pattern was observed in A. toltecus, while in $G$. soricina the second period begins later, in September-November in La Mina. The absence of lactating females of A. toltecus and $G$. soricina in La Mina, where the largest colonies are found (Table 1), indicates that births occur in one of the nearby tunnels between January and September as suggested (Webster \& Jones, 1982; Álvarez \& Álvarez-Castañeda 1991; Cloutier \& Thomas, 1992; Almazán-Catalán et al., 2015). Also, in these months the temperature and humidity in La Mina decrease below
$19{ }^{\circ} \mathrm{C}$, the humidity between $85-90 \%$ and the water level increases. Therefore, the availability of other nearby tunnels without water flow and with more suitable temperature and humidity (according to the best supported models, the probability of reproductive activity increases by three $(C$. perspicillata, $\mathrm{OR}=3.201)$, six $(G$. soricina, $\mathrm{OR}=6.147)$ and seven $($ A. toltecus, $\mathrm{OR}=7.777)$ times more when the temperature goes from $20-24{ }^{\circ} \mathrm{C}$ ). It offers them the opportunity to give birth and lactation in a safer way, since, unlike the sampled tunnel, does not present a current of water throughout the year, posing a danger to the young if they fall and die from drowning.

In the case of $G$. soricina, it has been reported that it is a species that can perch in a variety of sites and forms maternity colonies (Álvarez, Willing, Jones \& Webster, 1991; Uribe \& Arita, 2005). A colony may be in an abandoned house located $150 \mathrm{~m}$ from the sampled tunnel. The colony remained during the entire sampling period of this study; however, it was not possible to capture individuals and confirm that females go to that roost during birth and lactation. Unlike the tunnel with water flow, the abandoned house represents a better refuge for the growth of the offspring and against adversities.

The results of this study show that the reproductive cycles of the studied bat species are related to at least six variables (season, sex, roost, temperature, precipitation and humidity), highlighting the temperature, relative humidity and the roost site of the species. Future studies should increase the number of variables and include other variables, for example, weight, metabolic mass, or food availability, to help make more solid conclusions about the reproductive processes of Neotropical bat species. In addition, vaginal cytology studies can be complementary and generate more specific results of the time and duration of the reproductive patterns of bats.

Ethical statement: authors declare that they all agree with this publication and made significant contributions; that there is no 
conflict of interest of any kind; and that we followed all pertinent ethical and legal procedures and requirements. All financial sources are fully and clearly stated in the acknowledgements section. A signed document has been filed in the journal archives.

\section{ACKNOWLEDGEMENTS}

To the authorities of Pluma Hidalgo and Santa María Huatulco for their support and help during the study. To M. Calixto, M. Soriano, A. García. A. Matus, D. Peña, E. Ruiz, E. Caballero, J. Arellanes, J. López, L. Jiménez, L. Pacheco, M. Sánchez, and R. Prado for their valuable help during the field work. To the Instituto Politécnico Nacional de México for the financial support to conduct the study (projects SIP-20161645 and SIP-20171154 for the second author), and to the Mexican National Council for Science and Technology for the studentship awarded to the first author.

\section{RESUMEN}

Reproducción y dinámica poblacional de murciélagos cavernícolas en Costa de Oaxaca, México. Introducción: La reproducción es una fase crítica para la mayoría de los organismos vivos y en los murciélagos las estrategias reproductivas exhiben considerable complejidad y variabilidad. Objetivo: Describir los patrones reproductivos y la dinámica poblacional de siete especies de murciélagos: Artibeus toltecus, Carollia perspicillata, Glossophaga soricina, Mormoops megalophylla, Pteronotus fulvus, Pteronotus mesoamericanus y Natalus mexicanus, que se refugian en una mina (La Mina) y dos cuevas (El Apanguito y Cerro Huatulco), en el estado de Oaxaca, sureste de México. Métodos: el muestreo se realizó una vez al mes de julio 2016 a junio 2017. Los murciélagos se capturaron utilizando una trampa de arpa que fue colocada en la entrada de los refugios. La especie de los murciélagos capturados fue determinada con claves de identificación taxonómica, además fueron marcados con un anillo de aluminio y también se determinó el sexo y la clase de edad. La actividad reproductiva fue modelada a través de 63 GLMs para cada especie (504 en total). El mejor modelo fue seleccionado según el Criterio de Información de Akaike (AIC). Resultados: se capturaron y marcaron 5836 murciélagos, incluidos en 14 especies, 10 géneros y cinco familias. Las especies más abundantes fueron: $P$. fulvus y $P$. mesoamericanus, que representaron el 41 y el $32.3 \%$ de las capturas, respectivamente. Los mormópidos
M. megalophylla, P. fulvus, P. mesoamericanus junto con $N$. mexicanus mostraron un patrón monoéstrico estacional, mientras que los filostómidos A. toltecus, C. perspicillata y $G$. soricina mostraron un patrón poliéstrico estacional bimodal. La abundancia mensual de las especies fluctuó significativamente $(\mathrm{H}=13, \mathrm{df}=11, \mathrm{P}=0.044)$ en los tres refugios a lo largo del periodo de estudio y el GLM mejor respaldado que incluyó las siete especies mostró que las seis variables elegidas (temporada, sexo, refugio, temperatura, precipitación y humedad) se asociaron positivamente con la actividad reproductiva. Conclusiones: la actividad reproductiva de cada especie parece estar sincronizada con el final de la temporada seca y el comienzo de la temporada de lluvias, e influenciada por factores como temperatura, humedad y el sitio de refugio.

Palabras clave: cuevas; minas; monoéstrico; Mormoopidae; Phyllostomidae; poliéstrico; segregación sexual, México.

\section{REFERENCES}

Adams, J.K. (1989). Pteronotus davyi. Mammalian Species, 346, 1-5.

Almazán-Catalán, J.A., Sánchez-Hernández, C., RomeroAlmaraz, M.L., Sánchez-Vásquez, L., \& GonzálezPérez, S.B. (2015). Habitat use and reproduction of mammals from Tlaxmalac, at Balsas River basin, Guerrero, Mexico. The Southwestern Naturalist, $60(1), 36-44$

Altringham, J.D. (1996). Bats: Biology and Behavior. England: Oxford.

Álvarez, J., Willing, M., Jones, K., \& Webster, D. (1991). Mammalian Species, 379, 1-7.

Álvarez-Castañeda, S.T., Álvarez, T., \& González-Ruiz, N. (2015). Guía para la identificación de los mamíferos de México en campo y en laboratorio. México: Centro de investigaciones Biológicas del Noroeste, S. C. Asociación Mexicana de Mastozoología A.C.

Álvarez, T., \& Álvarez-Castañeda, S.T. (1991). Notas sobre el estado taxonómico de Pteronotus davyi en Chiapas y de Hylonycteris en México (Mammalia: Chiroptera). Anales de la Escuela Nacional de Ciencias Biológicas de México, 34, 223-229.

Anthony, E.L.P. (1988). Age determination in bats. In T.H. Kunz (Ed.), Ecological and behavioral methods for the study of bats (pp. 44-72). USA: Smithsonian Institution.

Ávila-Flores, R., \& Medellín, R. (2004). Ecological, taxonomic, and physiological correlates of cave use by Mexican Bats. Journal of Mammalogy, 85, 675-687.

Ayala-Téllez, H.L., Iñiguez-Dávalos, L.I., Olvera-Vargas, M., Vargas-Contreras, J.A.M., \& Herrera-Lizaola, 
O.A. (2018). Bats associated to caves in Jalisco, México. Therya, 9, 29-40.

Balmori, A. (1999). La reproducción en los quirópteros. Galemys, 11, 17-34.

Bateman, G.C., \& Vaughan, T.A. (1974). Nightly activities of mormoopid bats. Journal of Mammalogy, $55,45-65$.

Boada, C., Burneo, S., De Vries, T., \& Tirira, D. (2003). Notas ecológicas y reproductivas del murciélago rostro de fantasma Mormoops megalophylla (Chiroptera: Mormoopidae) en San Antonio de Pichincha, Ecuador. Mastozoología Neotropical, 10, 21-26.

Bonaccorso, F.J. (1979). Foraging and reproductive ecology in a Panamian bat community. Bulletin of the Florida State Museum of Biological Sciences, 24, 359-408.

Bonaccorso, F.J., Arends, A., Genoud, M., Canton, D., \& Morton, T. (1992). Thermal ecology of moustached and ghost-faced bats (Mormoopidae) in Venezuela. Journal of Mammalogy, 73, 365-378.

Bradbury, J.W., \& Veherencamp, S.L. (1976). Social organization and Foraging in emballonurid bats. Field Studies. Behavioral and Ecological Sociobiology, 1, 337-381.

Burland, T.I. (1998). Social organization and population structure in the brown long-eared bat Plecotus auritus (Doctoral thesis). University of Aberdeen, Scotland.

Clark, B.S., Leslie, D.M., \& Carter, T.S. (1993). Foraging activity of adult female Ozark big-eared bats (Plecotus townsendii ingens) in summer. Journal of Mammalogy, 74, 422-427.

Cloutier, D., \& Thomas, D. (1992). Carollia perspicillata. Mammalian Species, 417, 1-9.

Cumming, G.S., \& Bernard, R.T.F. (1997). Rainfall, Food abundance and timing of parturition in African bats. Oecología, 111, 309-317.

Dinerstein, E. (1986). Reproductive ecology of fruit bats and the seasonality of fruit production in a Costa Rican cloud forest. Biotropica, 18, 307-318.

Estrada, A., Coates-Estrada, R., \& Meritt, D. (1993). Bat species richness and abundance in tropical rain forest fragments and in agricultural habitats at Los Tuxtlas, México. Ecography, 16, 309-318.

Estrada, A., \& Coates-Estrada, R. (2001a). Species composition and reproductive phenology of bats in a tropical landscape at Los Tuxtlas, México. Journal of Tropical Ecology, 17, 626-646.

Estrada, A., \& Coates-Estrada, R. (2001b). Bat species richness in live fences and in corridors of residual rain forest vegetation at Los Tuxtlas, México. Ecography, 49, 213-225.

Fleming, T.H., Hooper, E.T., \& Wilson, D.E. (1972). Three Central American Bat communities: structure, reproductive cycles and movement patterns. Ecology, 53, 555-569.

Fleming, T.H. (1991). The relationship between body size, diet and habitat use in frugivorous bats, genus Carollia (Phyllostomidae). Journal of Mammalogy, 72, 493-501.

García-García, J.L., Santos-Moreno, A., \& RodríguezAlamilla, A. (2010). Population dynamics of bats Dermanura tolteca (Chiroptera: Phyllostomidae) in a tropical forest in México. Revista Biología Tropical, $58,1323-1334$.

Gobierno de México. (n.d.). Servicio Meteorológico Nacional. Retrieved from https://smn.conagua.gob.mx/es

Happold, D.C.D., \& Happold, M. (1990). Reproductive strategies of bats from Africa. Journal of Zoology, $222,557-583$.

Hill, J.E., \& Smith, J.D. (1984). Bats: a natural history. Austin, USA: University of Texas Press.

Humphrey, S.R., \& Bonaccorso, F.J. (1979). Population and Community ecology. In R.J. Baker, J.K. Jones Jr., \& D.C. Carter (Eds.), Biology of bats of the New World family Phyllostomatidae (pp. 409-441). USA: Special Publications of The Museum, Texas Tech University.

Iñiguez-Dávalos, L.I. (1993). Patrones Ecológicos en la comunidad de murciélagos de la sierra de Manantlán. In R.A. Medellín, \& G. Ceballos (Eds.), Avances en el estudio de los mamíferos de México (pp. 355-370). México: Asociación Mexicana de mastozoología A.C.

Jerrett, D.R. (1979). Female reproductive patterns in nonhibernating bats. Journal of Reproduction and Fertility, 56, 369-378.

Jiménez-Guzman, A., \& Ceballos, G. (2005). Pteronotus davyi. In G. Ceballos \& G. Oliva (Eds.), Los mamíferos silvestres de México (pp. 179-180). México: CONABIO.

Kunz, T.H. (1982). Ecology of bats. In T.H. Kunz (Ed.), Ecology of bats (pp. 1-55). New York, USA: Plenum press.

Kunz, T.H. (1996). Methods of marking bats. In D.E. Wilson, J. Nichols, R. Rudrin, R. Cole, \& M. Foster (Eds.), Measuring and monitoring Biological diversity standard methods for mammals (pp. 304-310). Washington, USA: Smithsonian Institution Press.

Lemos-Espinal, J.A., Rojas-González, R.I., Zúñiga-Vega, J., \& Jaime, J. (2005). Técnicas para el estudio de 
poblaciones de fauna silvestre. México: UNAM y CONABIO.

Medellín, R.A. (1993). Estructura y diversidad de una comunidad de murciélagos en el trópico húmedo mexicano. In R.A. Medellín, \& G. Ceballos (Eds.), Avances en el estudio de los mamíferos de México (pp. 353-354). México: Asociación Mexicana de Mastozoología, A.C.

Medellín, R., Arita, H., \& Sánchez, O. (2007). Identificación de los murciélagos de México, clave de campo (Segunda edición). México: Instituto de Ecología, UNAM.

Mello, M.A.R., \& Fernández, F.A.S. (2000). Reproductive ecology of the bat Carollia perspicillata (Chiroptera: Phyllostomidae) in a fragment of the Brazilian Atlantic coastal Forest. Zeitschrift für Säugetierkunde, 65, 340-349.

Mello, M.A.R., Schittini, G., Selig, P., \& Bergallo, H.G. (2004a). A test of the effects of climate and fruiting of Piper species (Piperaceae) on reproductive patterns of the bat Carollia perspicillata (Phyllostomidae). Acta Chiropterologica, 6(2), 309-318.

Mello, M.A.R, Schittini, G., Selig, P., \& Bergallo, H.G. (2004b). Seasonal variation in the diet of the bat Carollia perspicillata (Chiroptera: Phyllostomidae) in an Atlantic Forest area in southeastern Brazil. Mammalia, 68, 49-55.

OEIDRUS. (2005). Oficina Estatal de Información para el Desarrollo Rural Sustentable. Tarjeta municipal de información estadística básica.

R Core Team. (2020). R: A language and environment for statistical computing. Vienna, Austria: R Foundation for Statistical Computing. Retrieved from http:// www.R-project.org

Racey, P.A. (1978). The effect of photoperiod on the initiation of spermatogenesis in pipistrelle bats, Pipistrellus Pipistrellus. In R.J. Olembo, J.B. Castelino, \& F.A. Mutere (Eds.), Proceedings of the Fourth International Bat Research Conference. Kenya: Kenya Literature Bureau.

Racey, P.A. (1982). Ecology of bat reproduction. In T.H. Kunz (Ed.), Ecology of bats (pp. 57-104). NY, USA: Plenum Press.

Racey, P.A., \& Entwistle, A.C. (2000). Life-history and reproductive strategies of bats. In E.G. Crichton, \& P.H. Kruntzsch (Eds.), Reproductive Biology of Bats (pp. 363-414). London, England: Academic Press.

Ramírez-Pulido, J., Armella, M.A., \& Castro-Campillo, A. (1993). Reproductive patterns of three neotropical bats (Chiroptera: Phyllostomidae) in Guerrero, México. Southwestern Naturalist, 38, 24-29.
Rezsutek, M., \& Cameron, G.N. (1993). Mormoops megalophylla. Mammalian Species, 448, 1-5.

Sánchez-Hernández, C., Chávez-Tapia, C., Nunez-Garduno, A., Ceballos-Corona, E., \& Gurrola-Hidalgo, M.A. (1985). Notes on distribution and reproduction of bats from coastal regions of Michoacán, México. Journal of Mammalogy, 66(3), 549-553.

Simmons, N.B. (2005). Order Chiroptera. In D.E. Wilson \& D.M. Reeder (Eds.), Mammal species of the world: a taxonomic and geographic reference ( $3^{\text {rd }}$ Edition) (pp. 312-529). Baltimore, USA: Johns Hopkins University Press.

Thompson, S.D. (1992). Gestation and lactation in small mammals: basal metabolic rate and the limits of energy use. In T.F. Tomasi, \& T.H. Horton (Eds.), Mammalian Energetics: Interdisciplinary views of metabolism and reproduction (pp. 213-259). NY, USA: Cornell University Press.

Torres-Flores, J.W., López-Wilchis, R., \& Soto-Castruita, A. (2012). Dinámica poblacional, selección de sitios de percha y patrones reproductivos de algunos murciélagos cavernícolas en el oeste de México. Revista de Biología Tropical, 60, 1369-1389.

Torres-Flores, J.W., \& Santos-Moreno, A. (2017). Inventory, features, and protection of underground roosts used by bats in México. Acta Chiropterologica, 19, 439-454.

Trejo, I. (2004). Clima. In A.J. García-Mendoza, M.J. Ordóñez, \& M. Briones-Salas (Eds.), Biodiversidad de Oaxaca (pp. 67-85). México: Instituto de Biología, UNAM-Fondo Oaxaqueño para la Conservación de la Naturaleza-World Wildlife Fund.

Uribe, J., \& Arita, H. (2005). Glossophaga soricina. In G. Ceballos, \& G. Oliva (Eds.), Los mamiferos silvestres de México (pp. 217-218). México: CONABIO.

Webster, D., \& Jones, K. (1982). Artibeus toltecus. Mammalian Species, 178, 1-3.

Wilson, D.E. (1973). Reproduction in Neotropical bats. Periodicum Biologorum, 75, 215-217.

Wilson, D.E. (1979). Reproduction in Neotropical bats. In R.J. Baker, J.K. Jones Jr., \& D.C. Carter (Eds.), Biology of bats of the New World family Phyllostomidae (pp. 317-378). USA: Special Publications of The Museum, Texas Tech University.

Zortéa, M. (2003). Reproductive patterns and feeding habits of three nectarivorous bats (Phyllostomidae: Glossophaginae) from the Brazilian Cerrado. Brazilian Journal of Biology, 63, 159-168. 